A Finite Element Model for the simulation of the UL-94 burning test.

Julio Marti - Sergio R. Idelsohn - Eugenio Oñate

*Centre Internacional de Mètodes Numèrics en Enginyeria (CIMNE), Barcelona, Spain.

*Department of Civil and Environmental Engineering(DECA), Universitat Politècnica de Catalunya (UPC), 08034 Barcelona, Spain.

E-mail: julio.marti@cimne.upc.edu 
Noname manuscript No.

(will be inserted by the editor)

\title{
A Finite Element Model for the simulation of the UL-94 burning test.
}

Received: date / Accepted: date

\begin{abstract}
The tendency of the polymers to melt and drip when they are exposed to external heat source play a very important role in the ignition and the spread of fire. Numerical simulation is a promising methodology for predicting this behaviour. In this paper, an computational procedure that aims at analyzing the combustion, melting and flame spread of polymer is presented. The method models the polymer using a Lagrangian framework adopting the Particle Finite Element Method framework while the surrounding air is solved on a fixed Eulerian mesh. This approach allows to treat naturally the polymer shape deformations and to solve the thermo-mechanical problem in a staggered fashion. The problems are coupled using an embedded Dirichlet-Neumann scheme. A simple combustion model and a radiation modeling strategy are included in the air domain.
\end{abstract}

With this strategy the burning of a Polypropylene (PP) specimen under UL94 vertical test conditions is simulated. Input parameters for the modelling(density, specific heat, conductivity and viscosity ) and results for the validation of the numerical model has been obtained from different literature sources and by IMDEA burning a specimen of dimensions of $148 \times 13 \times 3.2\left[\mathrm{~mm}^{3}\right]$. Temperature measurements in the polymer have been recorder by means of 3 thermocouples exceeding the $1000[\mathrm{~K}]$. Simultaneously a digital camera was used to record the burning process. In adition, thermal decomposition of the material (Arrhenius coefficient $\mathrm{A}=7.14 \times 10^{16} \mathrm{~min}^{-1}$ and activation energy $\left.\mathrm{E}=240.67[\mathrm{Kj} / \mathrm{mol}]\right)$ as and changes in viscosity $(\mu)$ as a function of temperature were obtained. Finally, a good agreement between the experimental and the numerical can be seen in terms of shape of the polymer as well as in the temperature evolution inside the polymer.

Keywords Dripping, Melt flow, UL 94 Test, Particle Finite Element Method(PFEM)

Address(es) of author(s) should be given 
1 Introduction.

Notations
\begin{tabular}{|l|l|}
\hline Symbol & Parameter \\
\hline $3 \mathrm{D}$ & Three-dimensional \\
\hline$\Omega$ & Domain \\
\hline$\Gamma$ & Boundary \\
\hline $\mathrm{t}$ & Time \\
\hline $\mathbf{x}$ & Spacial position \\
\hline$\nabla$ & Nabla \\
\hline $\mathbf{v}$ & Velocity \\
\hline $\mathrm{p}$ & Pressure \\
\hline $\mathrm{T}$ & Temperature \\
\hline$\mu$ & Viscosity \\
\hline$\rho$ & Density \\
\hline $\mathbf{f}$ & Gravity force \\
\hline $\mathrm{C}$ & Heat capacity \\
\hline$\kappa$ & Thermal conductivity \\
\hline $\mathrm{I}$ & Spectral intensity \\
\hline s & Direction \\
\hline $\mathrm{c}$ & Speed \\
\hline$\epsilon$ & Emissivity \\
\hline $\mathrm{Y}$ & Mass fraction \\
\hline $\mathrm{w} T$ & Rate of production of heat \\
\hline
\end{tabular}

\begin{tabular}{|l|l|}
\hline $\mathrm{Q}_{\mathrm{R}}$ & Radiative heat flux \\
\hline$\epsilon_{\mathrm{V}}$ & Mass loss \\
\hline $\mathrm{Q}_{\mathrm{v}}$ & Heat absorbed \\
\hline$\alpha$ & Absorption coefficient \\
\hline$\sigma$ & StefanBoltzmann constant \\
\hline $\mathrm{G}$ & Incident radiation \\
\hline $\mathbf{q}$ & Heat flux \\
\hline $\mathrm{A}$ & Pre-exponential function \\
\hline $\mathrm{E}$ & Activation energy \\
\hline $\mathrm{T}_{\mathrm{a}}$ & Absolute temperature \\
\hline $\mathrm{R}$ & Universal gas constant \\
\hline $\mathrm{H}$ & Enthalpy of vaporization \\
\hline $\mathcal{K}$ & Bulk modulus \\
\hline $\mathbf{F}^{\mathbf{D}}$ & Drag force \\
\hline $\mathrm{C}_{\mathrm{D}}$ & Drag coefficient \\
\hline $\mathrm{A}_{\mathrm{CS}}$ & Cross sectional area \\
\hline $\mathrm{Subscripts}$ & \\
\hline $\mathrm{a}$ & Air \\
\hline $\mathrm{p}$ & Polymer \\
\hline $\mathrm{F}$ & Fuel \\
\hline $\mathrm{O}$ & Oxygen \\
\hline
\end{tabular}

Many of the objects that appear in modern household, workplaces and other consumer product areas are made of thermoplastic polymers. These range from mattresses, upholstered furniture to moulded objects, such as electronic products and fibre-reinforced composites.

Upon heating, thermoplastics suffer descomposition (pyrolysis) releasing combustible gases which mix with the oxygen of the ambient air to form an ignitable blend.

Having a sufficient concentratquantity of them, the ignition can take place either impulsively if the temperature is adequate for auto-ignition (the activation energy of the combustion reaction is attained) or due to the presence of an external source (flame or spark). Part of the heat released due to the combustion is fed back to the polymer causing further pyrolysis. If the evolved heat is sufficiently large to keep the decomposition rate of the polymer above the required limit to maintain the concentration of the volatiles within the flammability limits, a self sustaining combustion cycle is established.

From the above, it can be concluded that the combustion of polymers is similar to the combustion of many other solid materials. However, the tendency of polymers to spread the flame away from a fire source is a critical and singular aspect because many of them melt and tend to produce flammable drips or flow. Therefore, dripping is a big threat in polymer fires. It can accelerate the fire growth and spread the fire between nonadjacent objects producing secondary ignition.

Assessing the flammability of polymer materials is usually carried out via smallscale fire test. One of such test is the so-called vertical UL 94 test. In this test, a flame provided by a Bunsen is applied to a small-size specimen for 10 seconds forcing its ignition. After this period the flame is removed and the burning becomes free. This test is widely used for the quality control of polymeric products and the development of flame retardant materials. 
Modeling and computer simulation is another way of studying the polymers performance under fire conditions. Focusing on the condensed phase (polymer), considerable advances have been made in the modeling of pyrolysis phenomena responsible for thermal decomposition (and thus the burning rate) [1-3] and have become an integral part in existing fire simulation codes (e.g. [4], [5]). Basically, they solve transient conductive and radiative energy transfer coupled with decomposition chemistry [6]. These models are considerably complex and can potentially require a large number of input parameters (e.g. [7]) many of which cannot be known with high accuracy. Approaches have been developed in which these parameters are determined using inverse modeling coupled with evolutionary optimization algorithms $[8,9]$. The relationship between complexity and prediction error in the pyrolysis modelling have been explored in [10-13].

Although these account for different mechanisms affecting the degradation process, a phenomenon that is ignored is the melting of burning solid objects followed by melt flow and dripping phenomena in many references ( such as [14] and recent $[15-17]$. This means that it is assumed that surface regression does not occur, and the geometry/size of pyrolyzing or burning objects is invariant for the entire duration of the simulation which is appropriate for short duration simulations.

Regarding the numerical simulation of the UL94 test, the two strategies dealing with the problem at hand presented recently [18-20] ignored the computation of the gas flow and therefore the flame heat feedback was applied to the polymer as a boundary condition. Yet, the first one has shown the possibility of predicting the burning and melting of solid objects by including a continuum thermo-mechanical solid phase model in the calculations. Besides, in [19] the authors emphasize the fact that as polymer specimens are often ignited at corners, a three-dimensional burning model is more appropriate than a one or two dimensional model. Therefore, previously mentioned pyrolysis models could not be employed directly [5, $6]$.

Athough numerical simulation is a promissing methodology for predicting the behaviour, the uncertainty and lack of reliable input parameters for modeling the fire behavior of most materials is a challenge to tackle in fire simulations. Experimental characterization of several temperature-dependent material properties in fire situations has been recently done [21,22]. However, many of the necessary parameters are still not available (i.e.,properties at the temperature close to the decomposition). Adequate and reliable material properties at elevated temperatures (for different heating rates etc.) as input for thermally coupled models are mainly lacking, and even if available, stability and convergence is very difficult to achieve in practical fire simulations.

In this work we present improvement in the previously developed finite element thermo-mechanical model $[23,24]$ to analyze the melting of polymers under UL94 vertical test conditions. These include a model for the combustion, an improved radiation modeling strategy, an implicit solver for the motion of the air based on the solution of the compressible Navier-Stokes equations and a new version of Particle Finite Element Method (PFEM, www.cimne.com/pfem) to model the polymer motion. In the present approach the motion of the surrounding air is modeled solving a Eulerian formulation of the compressible Navier-Stokes equations equipped with the energy, radiation and combustion equations. On the other side, polymer motion and the evolution of the temperature are solved using the PFEM strategy [25]. Both thermo-mechanical problems are coupled using an embedded 
Dirichlet-Neumann scheme. With this strategy the burning of a Polypropylene (PP) specimen is simulated under UL94 vertical test conditions. The simulation results are analyzed and compared with the experimental data provide by IMDEA Materials Institute in Madrid(Spain).

\section{Governing equations}

This section is devoted to the mathematical formulation of the 3D heat transfer and fluid flow. Fig. 1 shows the geometry and the schematic diagram of the treated problem. The governing equations are considered to be three-dimensional, unsteady and compressible for the air while incompressible for the polymer. Different computational domains are used to represent the gas and the polymer. Thermo-physical and mechanical properties are assumed constant for both domain except for the viscosity of the polymer that depends on the temperature. The computation of the heat transfer and the fluid flow requires to solve simultaneously the Navier-Stokes and energy equations. Moreover, in order to take into account the radiation effects and the combustion process, the radiative transfer and species equations have to be solved only in the air domain. For futher details the reader is referred to [26-29].

Let $\Omega \subset \mathrm{R}^{3}$ be a bounded domain containing the polymer $\Omega_{\mathrm{p}}$ and the air $\Omega_{\mathrm{a}}$ which satisfy $\Omega_{\mathrm{a}} \cap \Omega_{\mathrm{p}}=\emptyset$ and $\Omega=\Omega_{\mathrm{a}} \cup \Omega_{\mathrm{p}}$ (see Fig.1). The subdomains are assumed to behave as viscous fluids ${ }^{1}$. We denote the time by $t$, the Cartesian spatial coordinates by $\mathbf{x}=\left.\mathrm{x}_{\mathrm{i}}\right|_{\mathrm{i}=1} ^{3}$, and the vectorial operator of spatial derivatives by $\nabla_{\mathrm{x}}=\left\{\partial_{\mathrm{x}_{\mathrm{i}}}\right\}_{\mathrm{i}=1}^{3}$. The evolution of the velocity $\mathbf{v}=\mathbf{v}(\mathbf{x}, \mathrm{t})$, the pressure $\mathrm{p}=\mathrm{p}(\mathrm{x}, \mathrm{t})$ and the temperature $\mathrm{T}=\mathrm{T}(\mathbf{x}, \mathrm{t})$ is governed by the following equation written in a compact form:

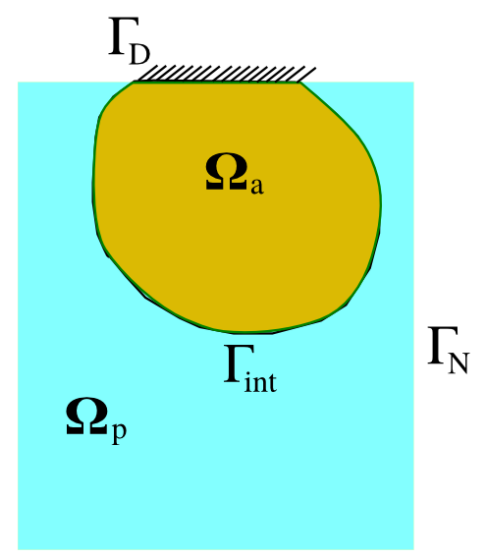

Fig. 1 Domain.

$$
\frac{\partial \rho \phi}{\partial t}+\nabla_{x} \cdot(\rho \mathbf{v} \phi)=\nabla_{x} \cdot\left(H_{\phi} \nabla_{x} \phi\right)+S_{\phi} \quad \text { in } \Omega \times(0, t)
$$

\footnotetext{
1 This assumption becomes satisfactory for higher temperatures and regarding a narrow range of shear rates like those experienced in the UL 94 test [30].
} 
In the following table $\phi, \mathrm{H}_{\phi}$ and $\mathrm{S}_{\phi}$ are summarized for the different variables:

\begin{tabular}{|l|c|c|r|}
\hline Transport of & $\phi$ & $H_{\phi}$ & $S_{\phi}$ \\
\hline Mass & 1 & 0 & $\epsilon_{v}(1.1)$ \\
\hline Momentum & $\mathbf{v}$ & $\mu$ & $-\nabla_{x} p+\frac{\mu}{3} \nabla_{x}\left(\nabla_{x} \cdot \mathbf{v}\right)+\rho \mathbf{f}(1.2)$ \\
\hline Energy & $T$ & $\kappa / C$ & $w_{T} / C+\left(\nabla \cdot Q_{R}\right) / C+Q_{v} / C(1.3)$ \\
\hline
\end{tabular}

where $\mu$ is the fluid viscosity, $\rho$ is the density, $p$ is the fluid pressure, $\mathbf{f}$ is the gravity force, $\mathrm{C}$ is the heat capacity, $\kappa$ is the thermal conductivity, $\mathrm{w}_{\mathrm{T}}$ is the rate of production of heat ${ }^{2}, \mathrm{Q}_{\mathrm{R}}$ is the radiative heat flux and $\epsilon_{\mathrm{v}}$ and $\mathrm{Q}_{\mathrm{v}}$ are the mass loss and heat absorbed due pyrolysis in the polymer, respectively. Note that for the polymer the terms $\mathrm{w}_{\mathrm{T}} / \mathrm{C}+\left(\nabla \cdot \mathrm{Q}_{\mathrm{R}}\right) / \mathrm{C}$ is set to zero. In addition, we shall assume for the air domain the ideal gas equation of state $\mathrm{p}=\rho / \mathrm{RT}$ which is the most common case for the compressible flows [31].

Equations (1.1-1.3) are completed with standard Dirichlet and Neumann boundary conditions. On the external boundary $\partial \Omega=\Gamma_{\mathrm{D}} \cup \Gamma_{\mathrm{N}}$, such that $\Gamma_{\mathrm{D}} \cap \Gamma_{\mathrm{N}}=\varnothing$

$$
\begin{gathered}
\left(\begin{array}{c}
\mathbf{v}=\overline{\mathbf{v}} \\
T=\bar{T}
\end{array}\right) \text { on } \Gamma_{D} \\
\left(\begin{array}{c}
\sigma . n=\bar{\sigma}_{n} \\
k \nabla T . n=\bar{q}_{n}
\end{array}\right) \text { on } \Gamma_{N}
\end{gathered}
$$

where $\overline{\mathbf{v}}$ and $\overline{\mathrm{T}}$ are the prescribed velocity and temperature respectively, $\mathbf{n}$ is the outer unit normal to $\Gamma_{\mathrm{N}}, \bar{\sigma}_{\mathrm{n}}$ and $\overline{\mathrm{q}}_{\mathrm{n}}$ are the prescribed traction vector and normal heat flux.

On the internal interfaces $\Gamma_{\text {int }}$, the coupling conditions are

$$
\begin{gathered}
\left(\begin{array}{c}
\llbracket \mathbf{v} \rrbracket=0 \\
\llbracket T \rrbracket=0
\end{array}\right) \text { on } \Gamma_{\text {int }} \\
\left(\begin{array}{c}
\llbracket \sigma \rrbracket . \mathbf{n}=0 \\
\llbracket k \nabla T \rrbracket \cdot \mathbf{n}=q_{R}
\end{array}\right) \text { on } \Gamma_{\text {int }}
\end{gathered}
$$

with $\mathbf{n}$ the unit normal to $\Gamma_{i n t}$, and $\llbracket \phi \rrbracket$ represents the jump of a quantity $\phi$ across the interface.

\section{Radiation modeling}

The effect of radiation is taken into consideration in the energy equation as the divergence of radiative heat flux i.e. $\nabla \cdot \mathbf{Q}_{R}$. Considering gray assumption and neglecting scattering, term $\nabla \cdot \mathbf{Q}_{\mathrm{R}}$ is given by

$$
\nabla \cdot \mathbf{Q}_{R}=\alpha\left(4 \sigma T^{4}-\int_{0}^{4 \pi} I(s) d s\right)
$$

where I is the spectral intensity at the time of the propagation along the direction $\mathrm{s}$ with speed $\mathrm{c}$ and $\alpha$ is the absorption coefficient. Eq.(6) requires the solution of the radiative transfer equations (RTE)

$$
\mathbf{s} \cdot \nabla I(s)=-\alpha I(s)+\alpha \frac{\sigma T^{4}}{\pi}
$$

\footnotetext{
2 Assuming constant values for the Schmidt $(\mathrm{Sc}=1)$ and Prandtl $(\mathrm{Pr}=1)$ numbers simplified composition and temperature dependent transport properties thus $\rho \mathrm{D}=\kappa / \mathrm{C}$
} 
Several methods can be used to solve Eq.(7) as by The Discrete Ordinate Method (DOM[32]) or the P1[29], among others. Adopting the P1 method, the intensity $I(s)$ is approximated as

$$
I(s)=\frac{1}{4 \pi}(G+3 \mathbf{q} \cdot \mathbf{s})
$$

where $G=\int_{0}^{4 \pi} I(s) d s$ is the incident radiation, and $\mathbf{q}$ the heat flux. Replacing Eq.(7) into Eq.(8) makes it possible to simplify the RTE equation so that the incident radiation is computed by solving the following system

$$
-\nabla \cdot\left(\frac{1}{3 \alpha} \nabla G\right)+\alpha G=4 \alpha \sigma T^{4}
$$

If the surfaces are taken to be diffuse and opaque it can be seen that ([29], p.515), under this approximation, the boundary condition becomes

$$
\frac{\partial G}{\partial n}=-\frac{3 \alpha \epsilon}{4-2 \epsilon} G+\frac{3 \alpha \epsilon}{4-2 \epsilon} 4 \sigma T^{4}
$$

where $\epsilon$ is the surface emissivity. Eq.10 is known as Marchak boundary condition.

The incident incident radiative heat flux to be prescribed onto the air-polymer interface $\Gamma_{\text {int }}$ (Eq. (5)) for the polymer is calculated as

$$
q_{R}=\frac{\epsilon}{2(2-\epsilon)}\left(4 \pi I_{b}-G\right)
$$

Note that radiation is only absorbed at the surface of the polymer as in previous works [30, 23, 18,20].

Accounting for the gasification effects

The mass loss $\left(\epsilon_{\mathrm{v}}\right)$ and heat absorbed $\left(\mathrm{Q}_{\mathrm{v}}\right)$ due to the pyrolysis process are modeled as:

$$
\begin{gathered}
\epsilon_{v}=-\rho A e^{-E / R T} \\
Q_{v}=\rho H \epsilon_{v}
\end{gathered}
$$

where $A$ is the pre-exponential function, $\mathrm{E}$ is the activation energy, $\mathrm{R}$ is the universal gas constant, $\mathrm{T}$ is the absolute temperature expressed in Kelvins and $\mathrm{H}$ is the enthalpy of vaporization. Note that both terms appear on the right hand side of Eqs.(1.1) and (1.3).

\section{Combustion model}

It is assumed that after pyrolysis the volatiles are released into the gas phase and combustion with air to form the flame immediately after they are generated. Following [33], the polymer/air reactive system is modeled as a simplified onestep chemical reaction between the fuel $(\mathrm{F})$ and the oxidizer $(\mathrm{O})$ to generate the product $(\mathrm{P})$. In particular the reaction is treated as $\mathrm{C}_{3} \mathrm{H}_{8}+5 \mathrm{O}_{2} \longrightarrow 3 \mathrm{CO}_{2}+4 \mathrm{H}_{2} \mathrm{O}$. For each specie $\mathrm{K}\left(\mathrm{C}_{3} \mathrm{H}_{8}, \mathrm{O}_{2}\right.$ and $\left.3 \mathrm{CO}_{2}+4 \mathrm{H}_{2} \mathrm{O}\right)$ the following equation is solved,

$$
\frac{\partial \rho Y_{K}}{\partial t}+\nabla \cdot\left(\rho \mathbf{v} Y_{K}\right)=\nabla\left(\kappa / C \nabla Y_{K}\right)+w_{k} / C ;
$$


The source terms are respectively $w_{C_{3} H_{8}}=-C B_{c} \rho^{2} Y_{F} Y_{O} \exp ^{\left(-T_{a} / T\right)}$ and $w_{O_{2}}=$ $-s w_{C_{3} H_{8}}$. In the air domain, the combustion heat release is introduced as $w_{T}=$

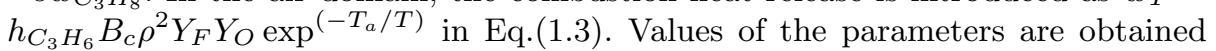
from [34]

\section{Numerical strategy}

3.1 Particle Finite Element method for the polymer

In the present work the polymer is modeled using the Particle Finite Element Method(PFEM). The PFEM uses an updated Lagrangian framework for describing the governing equations [35]. A finite element mesh discretized the domain where the equations are solved in the standard FEM methodology. Mesh nodes are treated as particles that can move freely and even separate from the main fluid domain according to their velocity. Due to the nodes motion, the finite element mesh is re-generated using Delaunay triangulation [36]. For further details on the PFEM approach the reader may refer to [25,37-39].

Assuming a nearly-incompressible behavior [40], the discrete version of the governing equations (Eq. 1.1-1.3) applying the standard backward Euler method and the Galerkin approach is

$$
\begin{gathered}
(\rho \mathbf{M}+\Delta t \mu \mathbf{K}) \mathbf{v}_{n+1}=\rho \mathbf{M} \mathbf{v}_{n}+\Delta t \mathbf{G} \mathbf{p}_{n}+\Delta t \rho \mathbf{F}+\Delta t \mathbf{F}^{D} \\
\mathbf{M} \mathbf{p}_{n+1}=\mathbf{M} \mathbf{p}_{n}-\Delta t \mathcal{K} \mathbf{D} \mathbf{v}_{n+1} \\
(\rho C \mathbf{M}+\Delta t \kappa \mathbf{K}) T^{n+1}=\rho C \mathbf{M} T^{n}+\Delta \mathbf{M} Q
\end{gathered}
$$

$\mathbf{F}^{\mathrm{D}}$ denotes the drag force resulting from the interaction between the air flow and the polymer particle:

$$
\mathbf{F}^{D}=\frac{1}{2} C_{D} A_{c s}\left\|v_{a}-v_{p}\right\|\left(v_{a}-v_{p}\right)(1 / \Omega) ;
$$

where $C_{D}$ is the drag coefficient, $v_{a}$ and $v_{p}$ are the air and polymer velocities respectively and $\mathrm{A}_{\mathrm{CS}}$ is the cross sectional area.

The mass $\mathbf{M}$, stiffness $\mathbf{K}$ and divergence $\mathbf{D}$ matrices are assembled from the elemental contributions as:

$$
\begin{gathered}
M_{i j}^{a b}=\delta_{i j} \int_{V} N^{a} N^{b} d V \\
K_{i j}^{a b}=\delta_{i j} \mu \int_{V} \frac{\partial N^{a}}{\partial x_{j}} \frac{\partial N^{b}}{\partial x_{j}} d V+\mu \int_{V} \frac{\partial N^{a}}{\partial x_{j}} \frac{\partial N^{b}}{\partial x_{j}} d V+ \\
\left(\Delta t \mathcal{K}-\frac{2}{3} \mu\right) \int_{V} \frac{\partial N^{a}}{\partial x_{i}} \frac{\partial N^{b}}{\partial x_{j}} d V \\
G_{i}^{a b}=\int_{V} \frac{\partial N^{a}}{\partial X_{i}} N^{b} d V
\end{gathered}
$$




$$
\begin{gathered}
f_{i}^{a}=\int_{V} N^{a} b_{i} d V \\
\mathbf{D}=\mathbf{G}^{T}
\end{gathered}
$$

where $\mathbf{N}$ are the standard linear FE shape functions [41], $V$ is the domain integration defined by the particles occupying the node position $\mathbf{x}$ at $t=t_{n+1}$ ( i.e. $\mathbf{x}_{n+1}$ ). The superscripts refer to the node indices while the Latin $(i$ and $j$ ) subscripts refer to the spatial components. The discrete operators follow the definitions given by Eqs. (19-23) which are calculated using the unknown configuration $\mathbf{x}_{n+1}$ according to the updated Lagrangian approach.

The governing system of equations (Eqs.15-17) is non-linear and the discrete operators must be updated at every non-linear iteration according to the newly obtained mesh position, which is usually evaluated as $\mathbf{x}^{n+1, i}=\mathbf{X}^{n}+\frac{\Delta t}{2}\left(\mathbf{v}_{n+1, i}+\right.$ $\mathbf{v}_{n}$ ). In order to avoid this iterative procedure, $\mathbf{x}_{n+1}$ is predicted using an explicit streamline integration. An extended description of this technique may be consulted in $[42,35,43]$. Once this algorithm is applied onto all the mesh nodes, the new configuration $V$ is obtained by creating a mesh connecting these nodes. Once the mesh is generated, the system (Eqs.15-17) is solved and the velocity $\mathbf{v}_{n+1}$, the pressure $p_{n+1}$ and the temperature $T_{n+1}$ are known.

\subsection{Finite Element formulation for the air}

The conservation Eqs.(1),(9) and (14) are discretized by a standard Eulerian FEM procedure with a Backward Euler scheme for the time integration([44], [45]). The equations to be solved in the air domain are:

$$
\begin{gathered}
\left(\mathbf{L}_{1 / 3 \alpha}+\mathbf{M}_{\alpha}\right) G=4 \alpha \sigma \mathbf{M}_{\alpha} T^{n+1,4} \\
\left(C \mathbf{M}+\Delta t C \mathbf{C}\left(\mathbf{v}^{n}\right)+\Delta t \mathbf{L}_{\kappa / \rho}\right)^{n+1}=C \mathbf{M} T^{n} \\
\Delta t \mathbf{M}_{1 / \rho} w_{T}+4 \alpha \sigma \Delta t \mathbf{M}_{1 / \rho} T^{n+1,4}-\Delta t \mathbf{M}_{1 / \rho} G \\
\left(\mathbf{M}+\Delta t C \mathbf{C}\left(\mathbf{v}^{n}\right)+\Delta t \mathbf{L}_{\kappa / \rho}\right) Y_{k}^{n+1}=C \mathbf{M} Y_{k}^{n}+\Delta t \mathbf{M}_{/ \rho} w_{k} \\
\left(\mathbf{M}+\Delta t \mathbf{C}(\mathbf{v})+\Delta t \mathbf{K}_{\mu / \rho}\right) \mathbf{v}^{n+1}=\mathbf{M} \mathbf{v}_{n}+\Delta t \mathbf{G}_{1 / \rho} \mathbf{p}_{n+1}+\Delta t \mathbf{F} \\
\mathbf{M}_{R T / \rho}\left(\frac{p^{n+1}-p^{n}}{\Delta t}\right)+\mathbf{D} \mathbf{v}^{n+1}=\mathbf{0}
\end{gathered}
$$

Matrix sub-indeces denote that the correspondly elemental contributions are multiplied by the value of the sub-index evaluated at the Gauss point of the element. Eqs.(25-28) are further stabilized in space using an Algebraic Sub-Grid Scales (ASGS) technique [46]. A fractional step procedure ([47], [48], [49], [50] or [51]) is used for the solution of Eqs.(27) and (28). 
The discrete operators that correspond to the terms entering the Galerkin part of the weak form are

$$
\begin{gathered}
\mathbf{M}=\sum_{i=1}^{\text {element }} \int_{V} N^{a} N^{b} d V_{i} \\
\mathbf{G}=\sum_{i=1}^{\text {element }} \int_{V} \nabla N^{a} N^{b} d V_{i} \\
\mathbf{L}=\sum_{i=1}^{\text {element }} \int_{V} \nabla N^{a} \nabla N^{b} d V_{i} \\
\mathbf{C}(\mathbf{v})=\sum_{i=1}^{\text {element }} \int_{V} N^{a} \mathbf{v} \cdot \nabla N^{b} d V_{i} \\
\mathbf{F}=\sum_{i=1}^{\text {element }} \int_{V} N^{a} F d V_{i}
\end{gathered}
$$

Eqs $(25,27-28)$ represents the implicit version of the explicit strategy presented by the authors in $[23,52]$. While in an explicit scheme the density $\rho^{n}$ is known during the time step, here is unknown and needs to be reevaluated in every iteration. At the beginning of each time step the density is initialized as $\rho^{n+1}=\rho^{n}$ and is subsequently updated with the last value of the nonlinear iteration.

\section{Overall strategy}

The overall solution of the problem involving thermal and mechanical interaction of the polymer and the air can be summarized in the box below

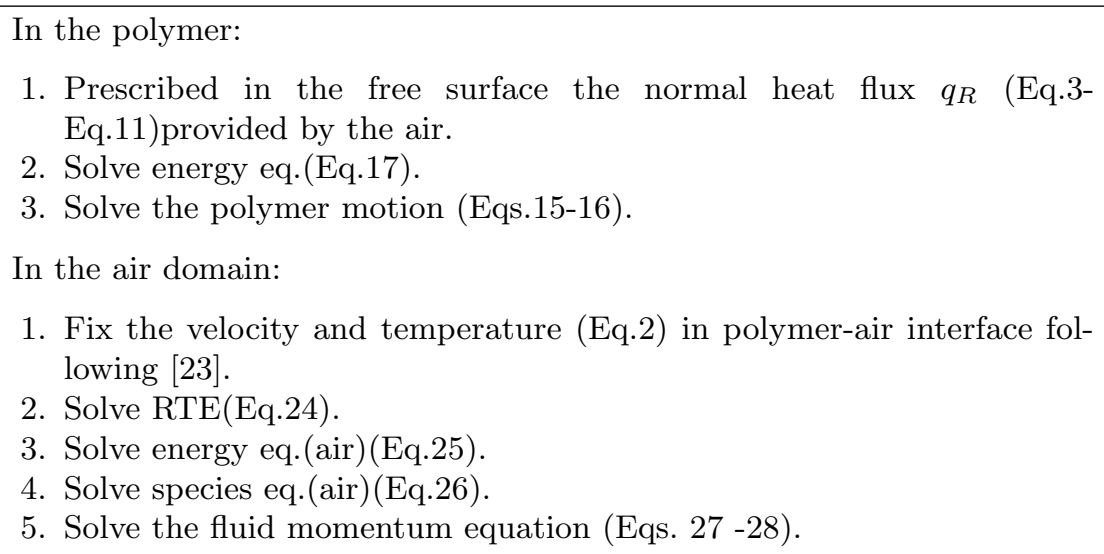

1. Fix the velocity and temperature (Eq.2) in polymer-air interface following [23].

2. Solve RTE(Eq.24).

3. Solve energy eq.(air)(Eq.25).

4. Solve species eq.(air)(Eq.26).

5. Solve the fluid momentum equation (Eqs. $27-28$ ). 
5 Validation model and numerical computation

The burning of a polymer in the UL 94 set up has been modeled numerically with the strategy proposed in this work. The numerical scheme has been implemented in the open source code KRATOS ([53]). Next we present some details about how the PP specimen was prepared as important parameters and data for the numerical simulation and posteriorly validation by IMDEA Materials Institute. Finally, details of the numerical set up as well as numerical results are presented.

\section{Material and method}

The PP specimen (Repsol PP ISPLEN 045 G1E [54]) was prepared using a twin-screw extruder (KETSE 20/40 EC, Brabender) at 200 $\left.{ }^{\circ}\right]$ and the velocity was $60[\mathrm{rpm}]$. The extruded strands were cut into pellets and then the PP composites were injected with an injection molding machine (Arburg $320 \mathrm{C}$ ) for further tests. Complex viscosity of the PP measured with a rheometer (AR2000EX, TA Instruments) at the mode of dynamic temperature step and dynamic frequency sweeping of $0.1[\mathrm{rad} / \mathrm{s}]$, is reported in Fig.2.

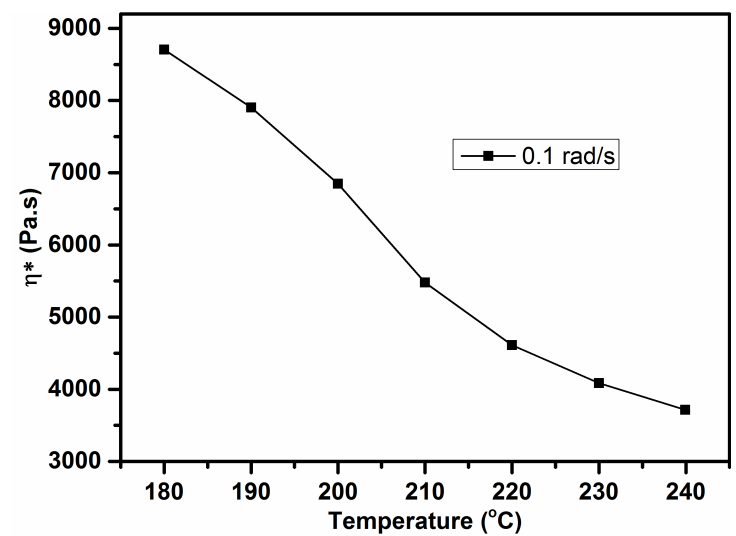

Fig. 2 Curve of viscosity

To characterize the thermal degradation and calculate the Arrhenius coefficient and the activation energy a thermogravimetric analysis has been carried out in air using a TGA Q50. The samples were heated with four different heating rates of $2,5,10,15\left[{ }^{\circ} \mathrm{C} / \mathrm{min}\right]$ and the weight loss was measured. The kinetic parameters were obtained by methods of Kissinger [55] and Flynn Wall Ozawa (FWO) $[56,57]$. Activation energy and pre-exponential for the first method are 240.67 $[\mathrm{KJ} / \mathrm{mol}]$ and $7.14 \times 10^{16}\left[\mathrm{~min}^{-1}\right]$ while for the second one are $245.62[\mathrm{KJ} / \mathrm{mol}]$ and $1.54 \times 10^{16}\left[\mathrm{~min}^{-1}\right]$.

Values for the thermo-physical properties of $\rho=905.0\left[\mathrm{~kg} / \mathrm{m}^{3}\right]$ [54], $\kappa=0.16$ $[\mathrm{W} / \mathrm{mK}]$ and $\mathrm{C}=1920[\mathrm{~J} / \mathrm{kgK}]$ are used as default [58].

A vertical burning test instrument(FTT, U.K.) was used to conduct the test with sheet dimensions of $148 \times 13 \times 3.2\left[\mathrm{~mm}^{3}\right]$ according to ASTM D3801. A flame was applied at the bottom of the specimen at an angle of $45\left[^{\circ}\right]$.

During the combustion, the temperature was measured and monitored in the condensed phase with three thermocouples K-type with $0.75[\mathrm{~mm}]$ nominal diam- 
eter and $500[\mathrm{~mm}]$ long-Incomel 600 from [59] embedded in the polymer. This miniature semi rigid thermocouple was selected due to the response, size, displacement and robustness of the assembly. Moreover, this type of thermocouples is recommended for the cases where the temperature changes are rapid as the presented in this work. More details are provided in [59]. In Fig.4 one can see how the instrumentation was positioned and fixed. The real time/temperature data were recorded through a data acquisition apparatus at $0.5[\mathrm{~s}]$. Simultaneously a digital camera was used to record the burning process.

\section{Numerical setup and results}

In this work, only a fourth part of the polymer is analyzed assuming symmetrics conditions as in [19]. In the symmetry faces slip and adiabatic boundary conditions are imposed(Fig.3(a)). The clamping of the sample is modelled by fixing the velocities to zero on the top of the specimen. The domain is discretised by 100,902 four-noded tetrahedra(see Fig.3(b)). For the air domain, velocity should not be imposed in the inlet since hot temperature in the vicinity of the flame will increase the buoyancy force and increase locally the air velocity. However such boundary condition would lead to creation of velocities in some parts of the bottom boundary pointing in the opposite direction(downwards) which leads to divergence of the numerical solver. With the aim of correcting this problem, a constant uniform velocity is prescribed at the inlet $\left(\overline{\mathbf{v}}_{\mathrm{y}}=0.2[\mathrm{~m} / \mathrm{s}]\right)$. While at the vertical walls slip and adiabatic boundary condition are applied, the top boundary is defined as a pressure-outlet.

A non-uniform triangular mesh (see Fig.3(b)) formed by 286,850 tetrahedra discretizes the air domain.

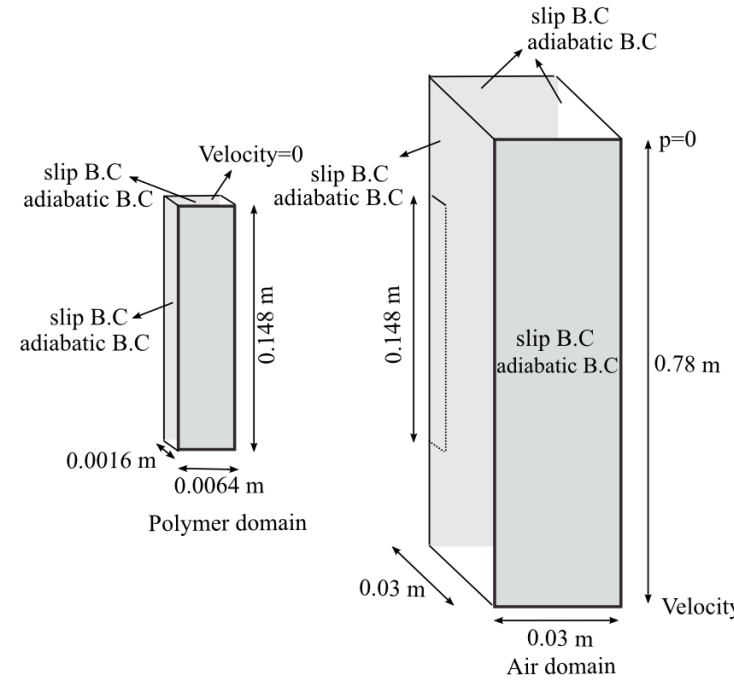

(a) Domains and boundaries conditions

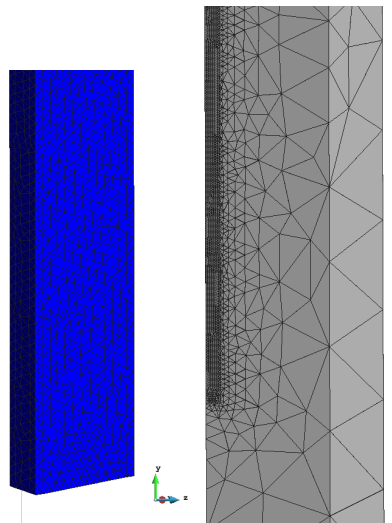

(b) Polymer and air meshes

Fig. 3 Problem definition. 


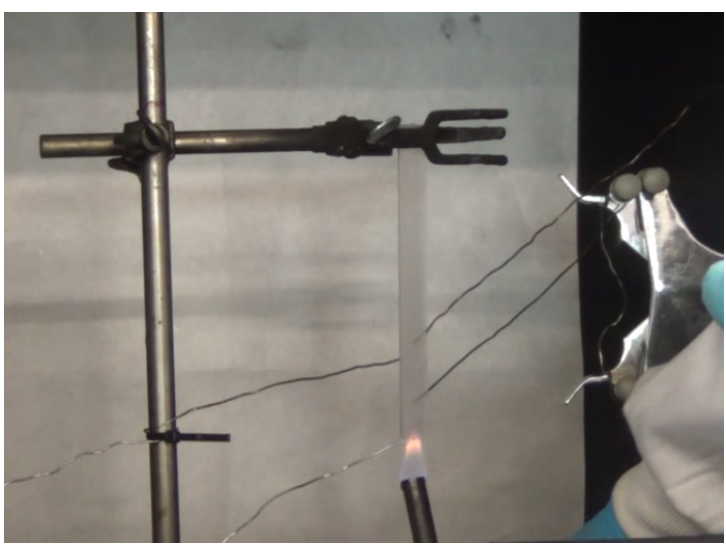

(a) View of the set-up.

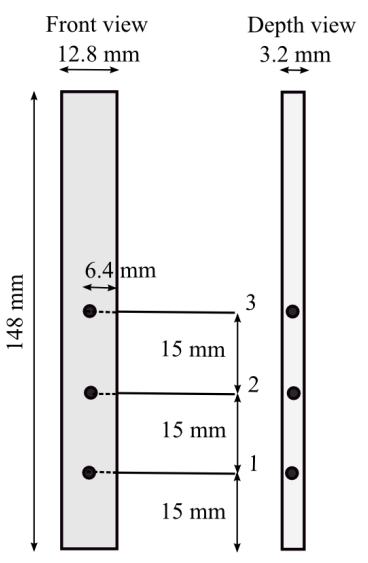

(b) Position of the thermocouples.

Fig. 4 Position of the thermocouples

The heat flux of the burner in the UL 94 test is applied as an external constant heat flux of $85\left[\mathrm{~kW} / \mathrm{m}^{2}\right]$. This is an average value obtained from [19]. The heat flux applied following the criteria presented in[18] during the first $10[\mathrm{~s}]$. Initially, the gas temperature is set to 298 [K]. The initial fuel and oxygen mass fractions are 0.0 and 0.23 , respectively. Once the sample surface reaches the decomposition temperature $650[\mathrm{~K}][60]$, the value of $\mathrm{Y}_{\mathrm{F}}$ and $\mathrm{Y}_{\mathrm{O}}$ are fixed to 1 and 0 respectively. Regarding thermo-mechanical properties, in the gas phase thermal conductivity $\kappa$, the density $\rho$ and the specific heat $\mathrm{C}$ are set up to $0.0131[\mathrm{~W} / \mathrm{mK}], 1.0\left[\mathrm{~kg} / \mathrm{m}^{3}\right]$ and $1310.0[\mathrm{~J} / \mathrm{kgK}]$ [34], respectively while the values of these properties for the polymer are chosen to be $0.16[\mathrm{~J} / \mathrm{kgK}], 905.0\left[\mathrm{~kg} / \mathrm{m}^{3}\right]$ and $1920.0[\mathrm{~W} / \mathrm{mK}]$ respectively. Note that density changes are described by the ideal gas equation presented in section 2. The problem was simulated during $100[\mathrm{~s}]$. The input parameters are summarized in Table 1.

The viscosity in the air is considered constant while in the polymer it is a function of temperature. The analytical definition of this curve (see Fig.2 ) corresponding to temperatures between $180\left[{ }^{\circ} \mathrm{C}\right]$ and $270\left[{ }^{\circ} \mathrm{C}\right]$ is given next.

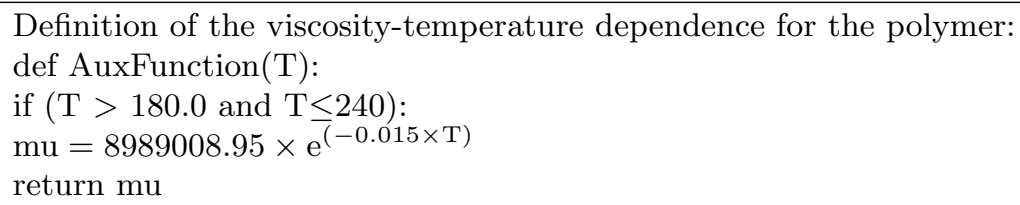

For the cases of temperatures below $180\left[{ }^{\circ} \mathrm{C}\right]$ and above $270\left[{ }^{\circ} \mathrm{C}\right]$ see Fig. 2, viscosity is approximated using the curve provided by NIST and published in [30].

The computed temperature distribution for different time steps across three horizontal cut planes at different heights of the air domain is presented in Fig.5(a). For the same time steps, the distribution of the fuel is shown in Fig.5(b). The presence of fuel is limited to a very thin layer around the polymer-air interface. This is due to the fact that convective transport is more important than the 
diffusive one as a result of the buoyancy forces. Fuel mix with the oxygen and react with the formation of a flame (see Fig. 5(a)).

From the Figs. it is seen that the heat zone evolves in the air domain giving heat feedback to the polymer. As time progresses the viscosity value decreases by several orders of magnitude in the polymer. As consequence of the viscosity decrease the sample experiences volume reduction due to melting and dripping together with gasification. This starts after a heating time of about $20[\mathrm{~s}]$ and can be seen in detail in Fig.6.

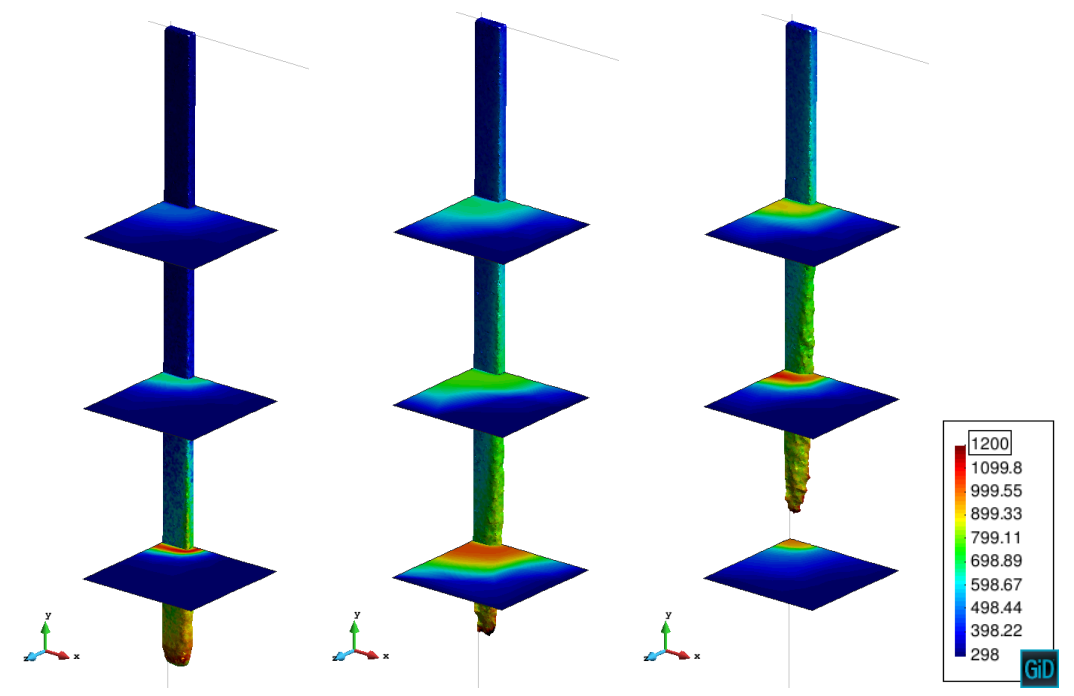

(a) Temperature distribution
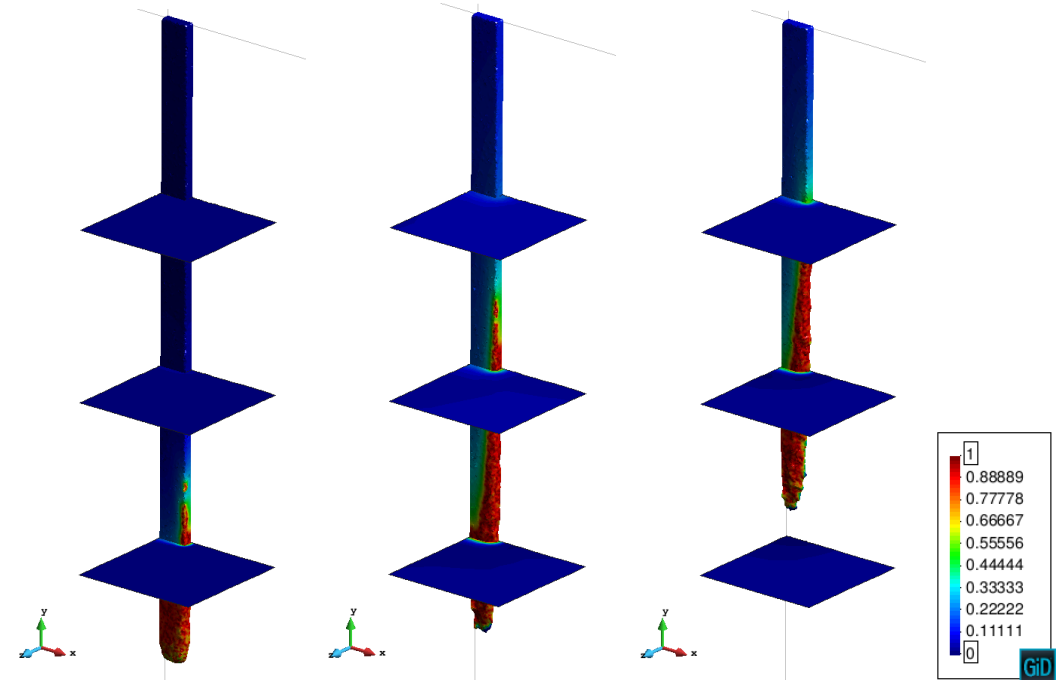

(b) Fuel distribution

Fig. 5 Evolution of the temperature and fuel distribution at time 10, 30 and $50[\mathrm{~s}]$. 


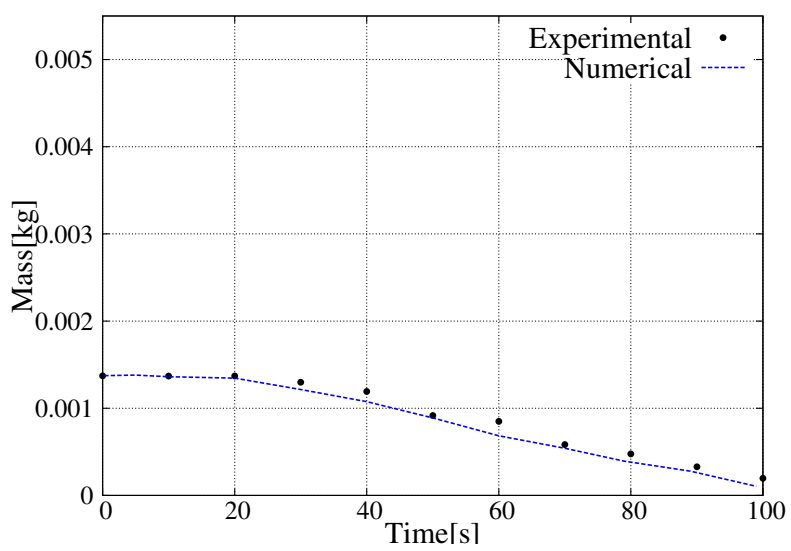

Fig. 6 Mass vs time for polymer in sample.

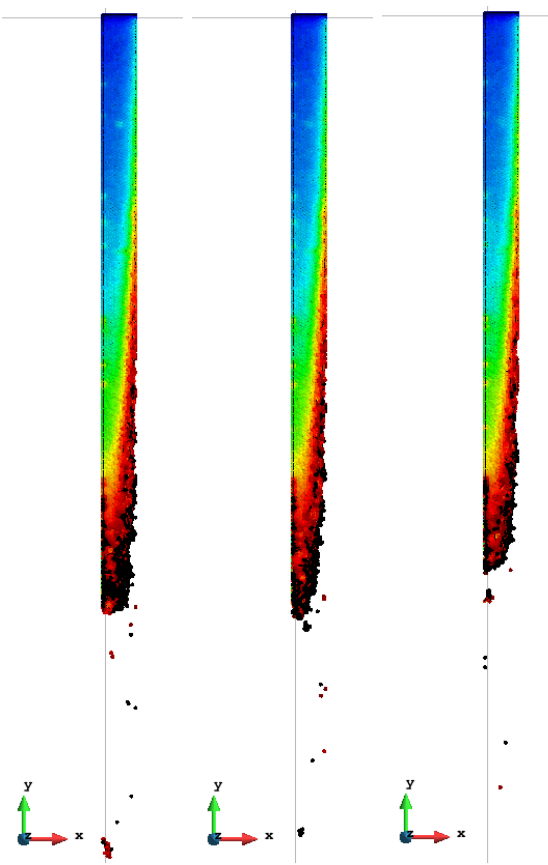

Fig. 7 Evolution of the melt flow at 40,43 and 47 [s]

Figs. 8(a)-8(b) show a comparison between the experimental and numerical results between 0 and $100[\mathrm{~s}]$. In these Figs., the original domain of the polymer is indicating in dotted lines. A good correlation in the shapes of the specimen between the numerical and the experimental results can be observed up to 60 [s]. From this moment, the polymer begins to drip continuously to form a thread of molten material that PFEM cannot capture. Besides, it is seen that the temperature at 
the edges is always higher than at the surface center, which is probably due to the edge effect $[61,62]$. Details of the evolution of the free surface and the melting and dripping can be seen in Fig.7.

Table 1 Input parameters for the Eulerian and PFEM solvers.

\begin{tabular}{l|c|c} 
Parameter & Air & Polymer \\
\hline Density & see Section 2 & $905\left[\mathrm{~kg} / \mathrm{m}^{3}\right]$ \\
Thermal conductivity & $0.0131[\mathrm{~W} / \mathrm{mK}]$ & $0.16\left[\mathrm{~W} / \mathrm{mK}^{2}\right]$ \\
Specific heat capacity & $1310.0[\mathrm{~J} / \mathrm{kgK}]$ & $1920.0[\mathrm{~J} / \mathrm{kgK}]$ \\
Viscosity & $1 \times 10^{-5}\left[\mathrm{~m}^{2} / \mathrm{s}\right]$ & $\mu(\mathrm{T})$ \\
Emissivity & - & 1.0 \\
StefanBoltzmann constant & $5.67 \times 10^{-8}\left[\mathrm{~W} / \mathrm{m}^{2} \mathrm{~K}^{4}\right]$ & - \\
Arrhenius coefficient & - & $7.14 \times 10^{16}\left[\mathrm{~min}^{-1}\right]$ \\
Activation energy & - & $240.67\left[\mathrm{KJ} / \mathrm{mol}^{-1}\right]$ \\
Universal gas constant & $8.3144[\mathrm{~J} / \mathrm{Kmol}]$ & - \\
Enthalpy of vaporization & - & - \\
$\mathrm{B}_{\mathrm{c}}$ & $5.96 \times 10^{9}\left[\mathrm{~m}^{3} / \mathrm{kgs}\right]$ & - \\
$\mathrm{T}_{\mathrm{a}}$ & $10700[\mathrm{~K}]$ & - \\
$\mathrm{C}$ & $2.601 \times 10^{4}[\mathrm{Kj} / \mathrm{kg}]$ & -
\end{tabular}

In addition to losing mass due to melting and dripping, the polymer sample (main body) loses mass due to gasification. But both phenomena (gasification and melt flow and dripping) are strongly interrelated because the dripping changes the surface temperature of the sample and therefore affects the mass loss rate due to gasification as well. This characteristic makes it impossible to distinguish which was the mass loss resulting from gasification and which one was due to melting and dripping. Fig. 6 shows the comparison between the model and the experiment with respect to mass losses. Note that we plotted the mass corresponding to a fourth part of the polymer analyzed. The mass loss follows the same trend, although the numerical values are in some parts may differ by 10 percent from the experimental ones which is probably due to the differences in the evolution of the temperature in the polymer as it shown in Fig.9.

Figs. 9(a)-9(c) show a comparison between experimental and numerical results at different times for thermocouples 1,2 and 3 . One can see that as we move from sensor 3 in the direction of sensor 1 , the difference between the experimental and the numerical results becomes more evident. Probably, this could be due to some complex phenomena which occur during the burning of polymers, such as bubbling, char formation, micro-explosions, complex time-dependent decomposition, etc. that are not considered in this work. In addition, the temperature registered by sensor 1 is affected for the polymer drips passing trough it. The study of these phenomenas is clearly beyond the current modeling capabilities for burning samples and their investigation will be conducted in the future. However, despite of the fact that the correlation observed is not perfect, the simulation results and experimental values exhibit similar trends. Therefore, given complexity of the setup, we consider the agreement between the experiments and the simulation results to be satisfactory. Nevertheless, there is a need for a systematic assessment and improvement of existing sub-models and modelling practices as well as the determination of the input parameters which cannot be known with high accuracy. 

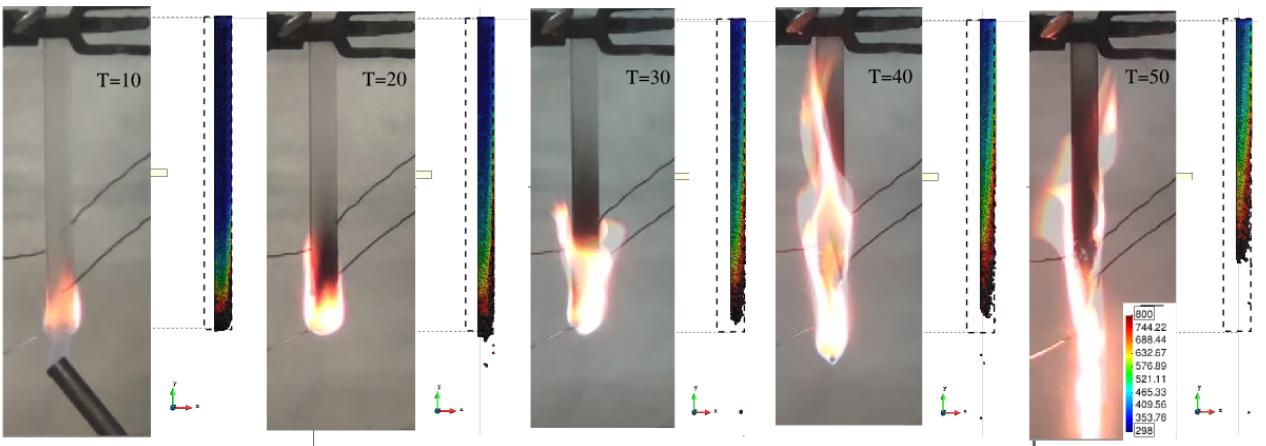

(a) $\mathrm{T}=10,20,30,40$ and $50[\mathrm{~s}]$.
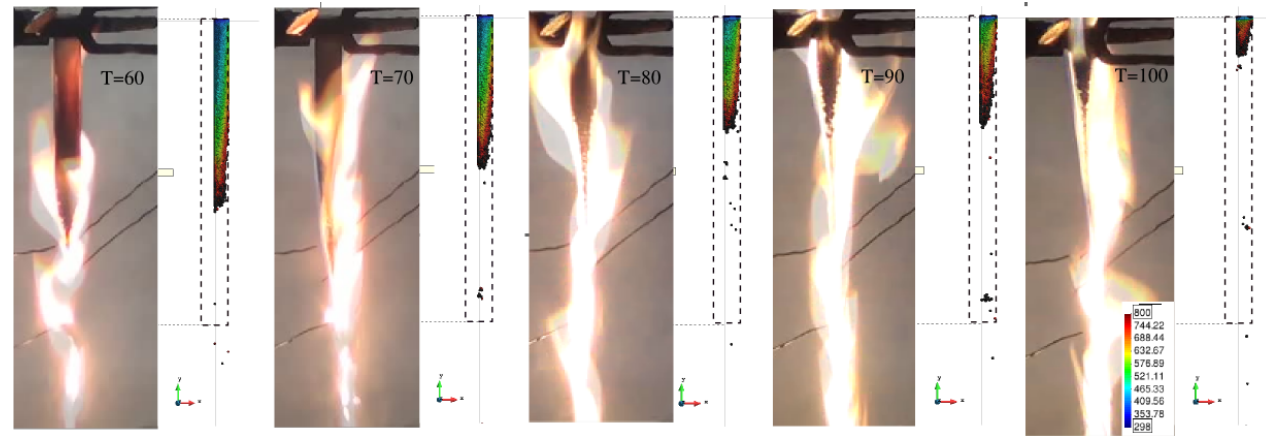

(b) $\mathrm{T}=60,70,80,90$ and $100[\mathrm{~s}]$

Fig. 8 Comparison between experimental versus numerical results.

\section{Conclusion}

The numerical strategy was developed for modeling of polymers in fire and used for reproducing the burning of UL94 test on a simple material (PP).

The numerical results show that the temperature agreed quite well the tendency of the temperature measurements given by the three thermocouples located inside the condensed phase. Consequently, during specimen combustion, PFEM's results show a good agreement with the experimental ones in terms of shape of the polymer evolution. Also, the mass loss follows similar trend, although the numerical values in some locations may differ from the experimental ones.

Although the model shows some deviation from the experimental results and needs further improvement in the future, one must note that unfortunally up-todate there exists no other numerical tool to simulate the melting and dripping of polymers and compare both results. In this sense, this work makes a new step in this direction as at least qualitatively the proposed strategy is capable of reproducing the test much more accurately than in the previous works dedicated to the same problem. Thus, the air modelling including a combustion and radiation models avoids to estimate a priori not only the value of the flame heat feedback but also where and how this need to be distributed along the polymer.

Although the model clearly requires several physical parameters to be determined, in order to "predict" a very well-known behavior, many of them have 


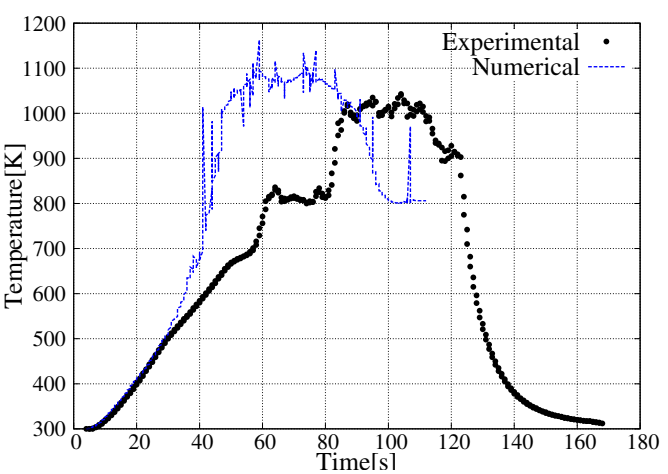

(a) Sensor 1

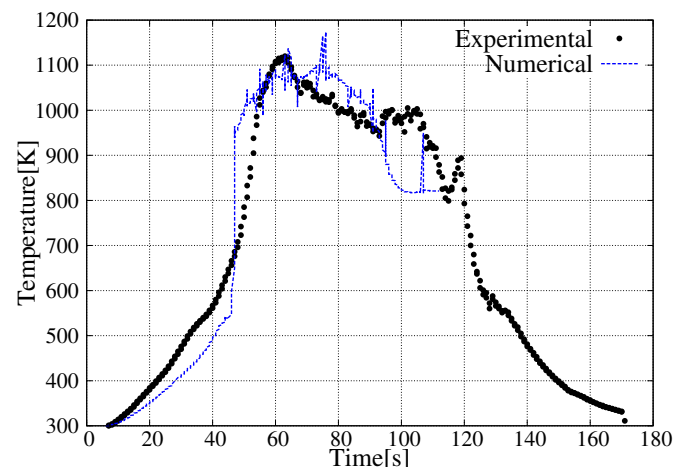

(b) Sensor 2

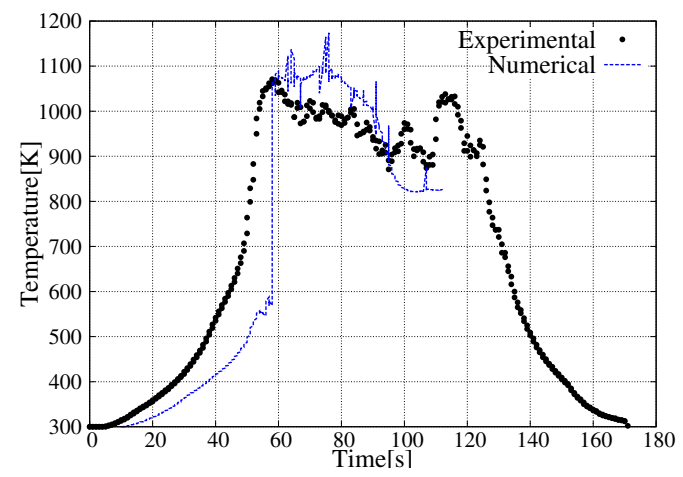

(c) Sensor 3

Fig. 9 Temperature evolution for sensors 1,2 and 3.

been obtained from different literature sources and diverse manufacturers. The temperature-dependent values determined for the viscosity of PP enabled PFEM to predict the dripping behavior in the UL 94 scenario. Nevertheless, a better characterization of the chemical reaction in the air and material properties as a function of temperature are needed to further improve the model. However, it is important to note that inclusion of temperature-dependent parameters is triv- 
ial inside our model. Therefore, in case of obtaining these dependencies from the experimentalists they can be immediately integrated in the model.

With this model the effects of model parameters on UL 94 test scenario could be studied through varying the value of a single parameter. Therefore, the importance of each parameter could be quantitatively evaluated. In addition, our numerical tool can be used for improving understanding of the complex burning behaviour including the dripping as the competition and interaction of gasification, flame inhibition and removal of fuel and heat from the pyrolysis zone by dripping.

In future work, it would be of interest to use the numerical tool for the prediction of flame spread and heat release rate in complex polymer geometries, modeling of standardized fire tests ranging from small scale tests to large-scale tests, and better understanding of flammability of anisotropic materials such as composites, and compare it with experiments carry out at larger scale.

\section{References}

1. Stoliarov S.I. and Lyon R.E. Thermo-kinetic model of burning for pyrolyzing materials. Fire Safety Science, page 11411152, 2008.

2. Lautenberger C. and Fernndez-Pello C. Generalized pyrolysis model for combustible solids. Fire Safety, 44:819839, 2009.

3. Lautenberger C. Gpyro3d: A three dimensional generalized pyrolysis model. Fire Safety Science, 11:193-207, 2014.

4. Firefoam code. http://www.fmglobal.com/modeling.

5. McGrattan K., McDermott R., Weinschenk C., and Forney G. Fire dynamics simulator (version 6), technical reference guide, 2013.

6. Stoliarov Stanislav I., Leventon Isaac T., and Lyon Richard E. Twodimensional model of burning for pyrolyzable solids. Fire and Materials, 38(3):391-408, 2013.

7. Lautenberger C. and Fernandez-Pello C. A model for the oxidative pyrolysis of wood. Combustion and Flame, 156(8):1503 - 1513, 2009.

8. Lautenberger C., Rein G., and Fernandez-Pello C. The application of a genetic algorithm to estimate material properties for fire modeling from bench-scale fire test data. Fire Safety Journal, 41(3):204 - 214, 2006.

9. Chaos M., Khan M., Krishnamoorthy N., de Ris J., and Dorofeev S. Evaluation of optimization schemes and determination of solid fuel properties for cfd fire models using bench-scale pyrolysis tests. Proceedings of the Combustion Institute, 33(2):2599-2606, 2011.

10. Ramroth W.T., Krysl P., and R.J. Asaro. Sensitivity and uncertainty analyses for fe thermal model of frp panel exposed to fire. Composites, Part A 37, 2006.

11. Linteris G.T. Numerical simulations of polymer pyrolysis rate: effect of property variations. Fire and Materials, 35:463480, 2011.

12. Stoliarov S.I., Safronava N., and Lyon R.E. The effect of variation in polymer properties on the rate of burning. Fire and Materials, 33:257271, 2009.

13. Bal N. and Rein G. Relevant model complexity for non-charring polymer pyrolysis. Fire Safety Journal, 61:36 - 44, 2013.

14. C.Di Blasi, S. Crescitelli, G. Russo, and G. Cinque. Numerical model of ignition processes of polymeric materials including gas-phase absorption of radiation. Combustion and Flame, 83(3):333 - 344, 1991.

15. Tsai T., Li M., Shih I., Jih R., and Wong S. Experimental and numerical study of autoignition and pilot ignition of pmma plates in a cone calorimeter. Combustion and Flame, 124(3):466 - 480, 2001.

16. Wu K., Fan W., Chen C., Liou T., and Pan I. Downward flame spread over a thick pmma slab in an opposed flow environment: experiment and modeling. Combustion and Flame, 132(4):697 - 707, 2003.

17. Gotoda H., Manzello S., Saso Y., and Kashiwagi T. Effects of sample orientation on nonpiloted ignition of thin poly(methyl methacrylate) sheets by a laser: 2. experimental results. Combustion and Flame, 145(4):820 - 835, 2006. 
18. Kempel F., Schartel B., Marti J., Butler K., Rossi R., Idelsohn S.R., Oñate E., and Hofmann A. Modelling the vertical ul 94 test: competition and collaboration between melt dripping, gasification and combustion. Fire and Materials, 39(6):570-584, 2015. FAM-14012 .

19. Wang Y., Jow J., Su K., and Zhang J. Development of the unsteady upward fire model to simulate polymer burning under ul 94 vertical test conditions. Fire Safety Journal, 54:1 13, 2012. Part of special issue: Large Outdoor Fires.

20. Matzen M., Marti J., Oñate E., Idelsohn S., and Schartel B. Advanced experiments and particle finite element modelling on dripping v-0 polypropylene, fire and materials 2017, 15th international conference, san francisco, ca, usa, 2017.

21. Li J. and Stoliarov S.I. Measurement of kinetics and thermodynamics of the thermal degradation for non- charring polymers. Combust. Flame, 160:1287-1297, 2013.

22. Stoliarov S.I., Safronava N., and Lyon R. E. The effect of variation in polymer properties on the rate of burning. Fire Mater, 33:257-271, 2009.

23. Marti J., Ryzhakov P., Idelsohn S., and Oñate E. Combined eulerian-pfem approach for analysis of polymers in fire situations. Int. J. Numer. Meth. Engng., 92:782-801, 2010.

24. Oñate E., Marti J., Ryzhakov P., Rossi R., and Idelsohn S. Analysis of the melting, burning and flame spread of polymers with the particle finite element method. Computer assisted methods in engineering and science, 20:165 - 184, 2013.

25. Idelsohn S., Oñate E., and Del Pin F. The Particle Finite Element Method: a powerful tool to solve incompressible flows with free-surfaces and breaking waves. International Journal of Numerical Methods in Engineering, 61:964-989, 2004.

26. Oliver X. and Agelet de Saracibar C. In Continuum Mechanics for Engineers. Theory and Problems. 2017.

27. Holzapfel G. In Nonlinear Solid Mechanics: A Continuum Approach for Engineering. Wiley, 2000

28. In Cox G., editor, Combustion Fundamentals of Fire. Academic Press, 1995.

29. Modest M.F. Radiative heat transfer. Academic Press, 2003.

30. Oñate E., Rossi R., Idelsohn S.R., and Butler K. Melting and spread of polymers in fire with the particle finite element method. International Journal for Numerical Methods in Engineering, 81:1046 - 1072, 2009.

31. Codina R., Vazquez M., and Zienkiewizc O.C. A general algorithm for compressible and incompressible flows. International Journal for Numerical Methods in Fluids, 27:13-32, 1998.

32. Fiveland W. The selection of discrete ordinate quadrature sets for anisotropic scattering, asme htd. fundam. radiat. Heat Transfer, 160:89-96, 1991.

33. Sousa Pessoa De Amorim M.T., Comel C., and Vermande P. Pyrolysis of polypropylene. Journal of Analytical and Applied Pyrolysis, 4(1):73 - 81, 1982.

34. Xie W. and DesJardin P. An embedded upward flame spread model using 2d direct numerical simulations. Combustion and Flame, 156(2):522 - 530, 2009.

35. Idelsohn S.R., Marti J., Limache A., and Oñate E. Unified lagrangian formulation for elastic solids and incompressible fluids: Application to fluidstructure interaction problems via the pfem. Computer Methods in Applied Mechanics and Engineering, 197(19):1762 1776, 2008. Computational Methods in FluidStructure Interaction.

36. Delaunay B. Sur la sphre vide. izvestia akademii nauk sssr. Otdelenie Matematicheskikh i Estestvennykh Nauk, 7:793-800, 1934.

37. Idelsohn S. R., Marti J., Souto-Iglesias A., and Oñate E. Interaction between an elastic structure and free-surface flows: experimental versus numerical comparisons using the pfem. Computational Mechanics, 43(1):125-132, Dec 2008.

38. Idelsohn S. R., Mier-Torrecilla M., Marti J., and Oñate E. The particle finite element method for multi-fluid flows. In Eugenio Oñate and Roger Owen, editors, Particle-Based Methods: Fundamentals and Applications, pages 135-158. Springer Netherlands, Dordrecht, 2011.

39. Oñate E., Idelsohn S. R., Celigueta M. A., Rossi R., Marti J., Carbonell J. M., Ryzhakov P., and Suárez B. Advances in the particle finite element method (pfem) for solving coupled problems in engineering. In Eugenio Oñate and Roger Owen, editors, Particle-Based Methods: Fundamentals and Applications, pages 1-49. Springer Netherlands, Dordrecht, 2011.

40. Kundu P.K. and Cohen I.M. Fluid mechanics. Academic Press, 2002.

41. Hughes T.J.R. The finite element method: Linear static and dynamic finite element analysis. Computer-Aided Civil and Infrastructure Engineering, 4(3):245-246, 1989. 
42. Idelsohn S.R., Nigro N., Gimenez J.M., Rossi R., and Marti J. A fast and accurate method to solve the incompressible navier-stokes equations. Engineering Computations, 30(2):197-222, 2013.

43. Ryzhakov P.B., Marti J., Idelsohn S.R., and Oñate E. Fast fluidstructure interaction simulations using a displacement-based finite element model equipped with an explicit streamline integration prediction. Computer Methods in Applied Mechanics and Engineering, $315: 1080-1097,2017$.

44. Donea J. and Huerta A. Finite element method for flow problems. Wiley, 2003.

45. Lohner R. Applied cfd techniques, 2nd edition. J. Wiley and Sons, 2008.

46. Codina R. A stabilized finite element method for generalized stationary incompressible flows. Computer Methods in Applied Mechanics and Engineering, 190(20-21):2681 - 2706, 2001.

47. Guermond J.L., Minev P., and Shen J. An overview of projection methods for incompressible flows. Computer Methods in Applied Mechanics and Engineering, 195:6011-6045, 2006.

48. Temam R. Sur lapproximation de la solution des equations de navier-stokes par la methode des pase fractionaires. Archives for Rational Mechanics and Analysis, 32:135-153, 1969.

49. Chorin A.J. A numerical method for solving incompressible viscous problems. Journal of Computational Physics, 2:12-26, 1967.

50. Ryzhakov P. A modified fractional step method for fluid-structure interaction problems. Revista Internacional de Métodos Numéricos para Cálculo y Diseño en Ingeniería, 33(12):58-64, 2017.

51. Ryzhakov P. and Marti J. A semi-explicit multi-step method for solving incompressible navier-stokes equations. Applied Sciences, 8(1), 2018.

52. Ryzhakov P., Rossi R., and Oñate E. An algorithm for the simulation of thermally coupled low speed flow problems. International Journal for Numerical Methods in Fluids, 70(1):119, 92012 .

53. Dadvand P., Rossi R., and Oñate E. An object-oriented environment for developing finite element codes for multi-disciplinary applications. Archieves of Computational Methods in Engineering, 17/3:253-297, 2010.

54. Repsol pp isplen 045 g1e. http://www.repsol.com/en/products-and-services/ chemicals.

55. Kissinger H. Variation of peak temperature with heating rate in differential thermal analysis. Journal of Research of the National Bureau of Standards, 57(4):217-221, 1956.

56. Flynn J. and Wall L. A quick, direct method for the determination of activation energy from thermogravimetric data. Journal of Polymer Science Part B:Polymer Letters, 4(5):323-328, 1966.

57. Ozawa T. A new method of analyzing thermogravimetric data. Bulletin of the Chemical Society of Japan, 38:1881-1886, 1965.

58. Thermal properties. http://www.engineeringtoolbox.com/.

59. Tc direct. http://www.tcdirect.es/.

60. Selected thermal properties. http://pslc.ws/fire/howwhy/thermalp.htm.

61. Wang Y., Zhang J., Jow J., and Su K. Analysis and modeling of ignitability of polymers in the ul-94 vertical burning test condition. J. Fire Sci., 27:561-581, 2009.

62. Wang Y., Zhang F., Jiao C., Jin Y., and Zhang J. Convective heat transfer of the bunsen flame in the ul94 vertical burning test for polymers. J. Fire Sci., 28:337-356, 2010. 